\title{
Combined processes of environmentally friendly technology for magnetic-pulse welding
}

\author{
Evgeny Strizhakov ${ }^{1}$, Stanislav Nescoromniy $^{1 *}$ \\ ${ }^{1}$ Don State Technical University, 1, Gagarin sq., Rostov-on-Don, 344000, Russia
}

\begin{abstract}
Various techniques for producing fixed joints in solid using electromagnetic fields are considered; basic diagrams, physics, features, and technical capabilities of each method are described. It is shown that thin-walled tubular irregular structures can be obtained under the magnetic-pulse moulding welding that joins the combined actions of induced currents passing through the overlap zone and magnetic pressure for apposing the weldable edges and for shaping in accordance with the matrix configuration. Obtaining joints from dissimilar materials and structures of different thicknesses is implemented due to shock pulse capacitor welding with magnetic pulse drive. The series connection of the weldable parts enables to synchronize the current flow and force impact on the weld junction. Depending on the combination of the weldable products, three techniques of shock pulse capacitor welding with magnetic pulse drive are proposed. To intensify the quality improvement of the female connectors obtained, it is proposed to use the magnetic-pulse welding in vacuum instead of the diffusion welding. Preheating of the complete unit in vacuum allows for the pre-activation of the connectable surfaces. A unique feature of the implemented diagram is a remote action on the telescopic joints of dissimilar alloys heated in vacuum to the pre-melting temperatures through a quartz glass.
\end{abstract}

\section{Introduction}

The application of electromagnetic pressure to intensify the processes of connecting materials in the solid phase is promising. A typical process of the magnetic-pulse welding (MPW) is carried out using a glancing collision that provides the appearance of a cleaning cumulative jet and a mutual deformation of the connectable juvenile surfaces. The process is similar to the explosion bonding, but a different energy source is used. Detonation in explosive is replaced by energy of the electromagnetic field [1]. Magnetic pulse welding (MPW) can produce high-quality connections of similar and dissimilar metals in the air or inert medium. Female closed-loop connections are mostly welded.

However, a typical MPW process cannot produce tubular irregular parts, connect core parts with sheet dissimilar materials, or obtain long joints of emission coatings with cathode bases.

Magnetic-pulse moulding welding, condenser magnetic hammer welding, and vacuumheat magnetic-pulse treatment can reduce the complexity of manufacturing thin-walled

\footnotetext{
*Corresponding author: nescoromniy@mail.ru
} 
2

closed-loop parts, dissimilar joints from parts of different thickness, and shell structures. All these advanced technologies are developed at Don State Technical University, Russia.

\section{Materials and Methods}

Magnetic-pulse moulding welding (MPMW).

As previously stated, the use of traditional magnetic-pulse welding to obtain thin-walled tubular parts according to the scheme with successive glancing collision is impossible. Magnetic-pulse treatment of parts with no closed loop has not been recommended at all until recently $[1,2]$.

The authors [2,3] propose a combined process of the magnetic-pulse moulding welding with the original gap. This made it possible to abandon the principle of glancing collision and to perform welding with induced currents passing through the overlap of the workpiece edges. Magnetic pressure is used as a welding and forming one.

Consequently, the processes of stamping and forming a welded joint are combined.

The electric resistance welding can be carried out in the atmosphere with the cleaning of the connectable surfaces by a splash of pollution, oxide and adsorbed films due to the electroerosion effect. The MPMW diagram is shown in Fig. 1. Conceptually, the combined process is as follows: the pre-cut thin sheet blank (1) is folded with overlap $(\Delta)$ and is set in matrix (2) with the original gap $(\mathrm{h})$, which has the shape of a future product. In the magneticpulse processing area, there is a work tool-inductor (3).

When the surge-current generator is discharged in the inductor (3), a high-frequency $(5 \div$ $100 \mathrm{kHz}$ ) electromagnetic field is created, under the action of which EMF of self-induction is induced in the blank (1) rolled up with overlap $(\Delta)$, an electric breakdown of the initial gap (h) occurs, and the electrical circuit breaks down. Through the blank (1), the induced current $\left(\mathrm{I}_{\mathrm{n}}\right)$ pulse flows, a pulsed arc arises in the gap, which heats and fuses the facing surfaces in the overlap zone. The force $\left(\mathrm{P}_{\mathrm{M}}\right)$ arising from the interaction of the induced current $\left(\mathrm{I}_{\mathrm{n}}\right)$ and the magnetic field $(\mathrm{H})$ of the inductor (3) deforms the blank towards the matrix (2). In this case, the insert that sizes up the gap (h) is knocked out; a layer of molten metal is squeezed out of the overlapping zone together with adsorbed and oxide films and other contaminants. Hot cleaned surfaces are compressed, pressed against the matrix, and the electric resistance welding is performed [4].

Magnetic pressure $\left(\mathrm{P}_{\text {magn }}\right)$ affects the entire workpiece area and performs molding in accordance with the matrix configuration. Thus, under the discharge of the surge-current generator with the duration of 50-200 $\mu$ s, the electric resistance welding and moulding of closed irregular structures from the sheet material occurs. The part is made from one setup. The loading diagram «for expansion», which enables to exclude distortion of thin-walled tubular parts, is carried out. 


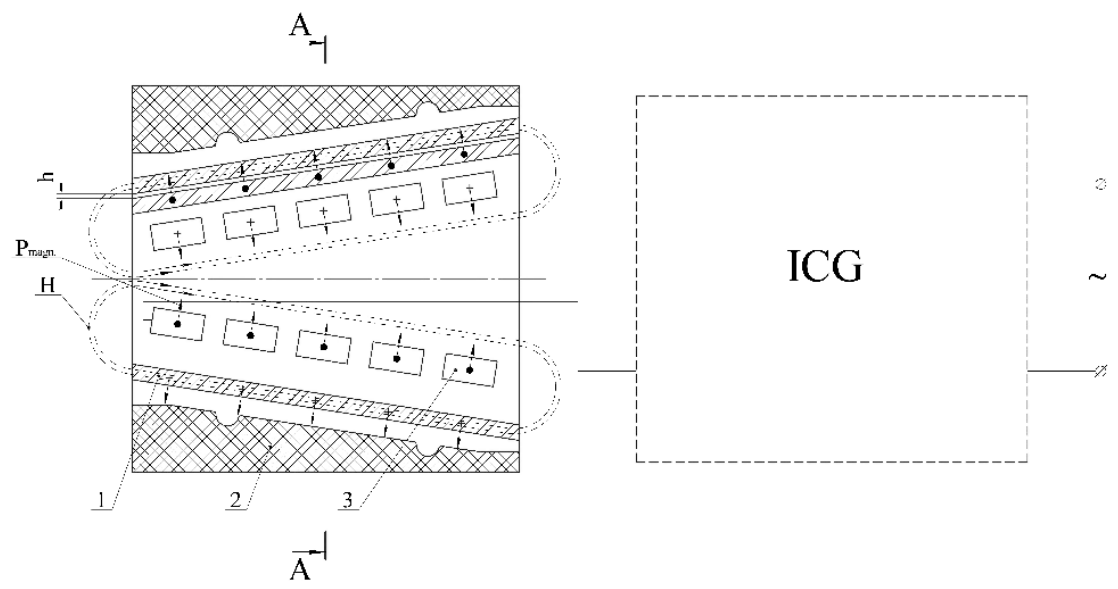

a) Process start-up

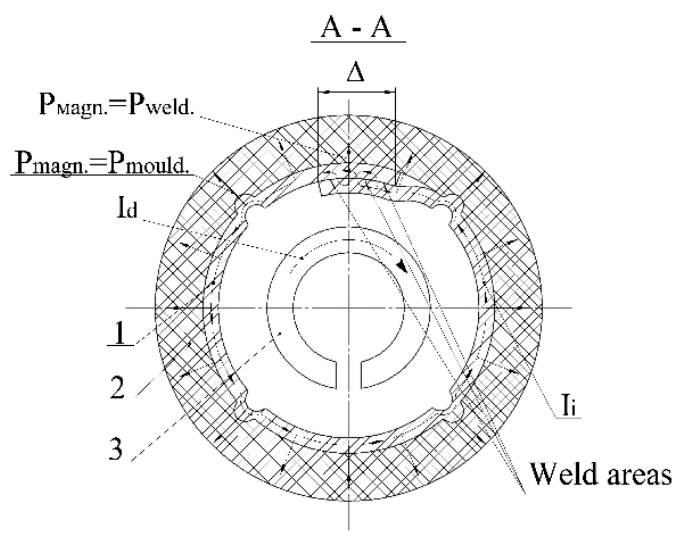

б) Process end

Fig. 1. Diagram of MPMW process: 1 is blank; 2 is matrix; 3 is inductor; $\mathrm{h}$ is original gap; $\Delta$ is overlap; ICG is impulse-current generator; $\mathrm{I}_{\mathrm{d}}$ is discharge current; $\mathrm{H}$ is magniflux; $\mathrm{I}_{\mathrm{i}}$ is induced current; $\mathrm{P}_{\text {magn. is magnetic pressure; }} \mathrm{P}_{\text {mould }}$ is moulding pressure; $\mathrm{P}_{\text {weld. }}$ is welding pressure

A short duration of the ICG discharge enables to narrow down the hot zone to welding temperatures to a limited extent necessary to provide sufficient strength of the connection. This allows welding on the parts with final processing (polished or with a decorative coating), providing the required marketable condition of the product. The advantages include the quality independence of the welded joint from the state of the connectable surfaces. The MPMW benefits include also the simplicity of the implementation of strength load and the absence of special displacement and upsetting devices.

The metallographic studies of weld junctions have not proved the formation of common grains; the weld junction is typical for welding in solid using the known pulsed welding 
4

methods. There is a clear interface line - a «setting» zone, similar to the explosion bonding and classical magnetic-pulse welding [5].

The authors, together with employees of Khrunichev Space Center, have developed the MPMW technology of lightweight stamp-welded shielding housings to wire the connector of the onboard cable networks of space vehicles. The $0,15 \div 0,2 \mathrm{~mm}$ thick aluminium foil shielding body is a typical tubular part of a complex shape. Lightweight housing is shown in Fig.2.

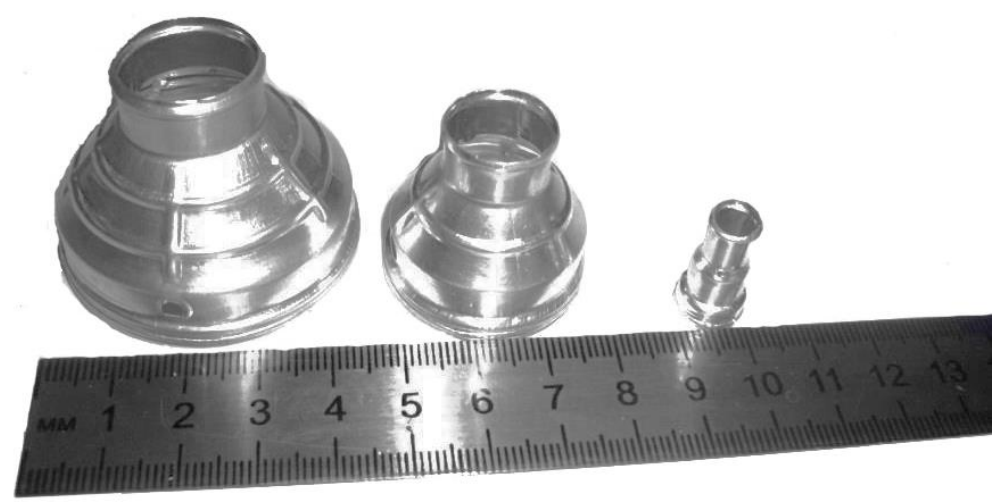

Fig. 2. Shielding housings of electric connectors of 3 standard sizes

Capacitor discharged percussion welding with magnetic pulse drive.

Making fixed joints from dissimilar materials and structures of different thicknesses always causes difficulties due to different conditions of heat removal under welding and to the appearance of intermetallic inclusions.

It is possible to solve the problem due to the use of pulse processes, in particular, highvoltage discharge of a capacitor bank.

The development of E.O. Paton Electric Welding Institute on the implementation of capacitor dischurged percussion welding (CDPW) with a spring drive [6] is known.

DSTU employees have proposed to use energy of the pulsed magnet fields to implement the forging force [7].

Induction-dynamic loading was produced using a magnetic pulse drive (MPD) consisting of an inductor and a pusher.

The basic diagram of the shock capacitor welding with MPD is shown in Fig. 3 [8].

The CDPW with MPD is as follows. The rod element is statically brought into contact (or installed with a preliminary gap) with a flat part and is connected in series with the MPD and ICG to synchronize the current flow and the force action on the weld junction.

When the ICG is discharged along the loops of the MPD inductor and 1 and 2 parts, a breakdown current pulse $\left(I_{d}\right)$ flows, whose alternating magnetic flux $(H)$ causes the occurrence of induced currents $\left(\mathrm{I}_{\mathrm{i}}\right)$ in the MPD pusher. The interaction of the magnetic field $(\mathrm{H})$ and the current $\left(\mathrm{I}_{\mathrm{i}}\right)$ in the pusher $(6)$ leads to the appearance of the magnetic pressure $\left(\mathrm{P}_{\text {magn }}\right)$, which affects the pusher that transmits the dynamic force to the rod element. 


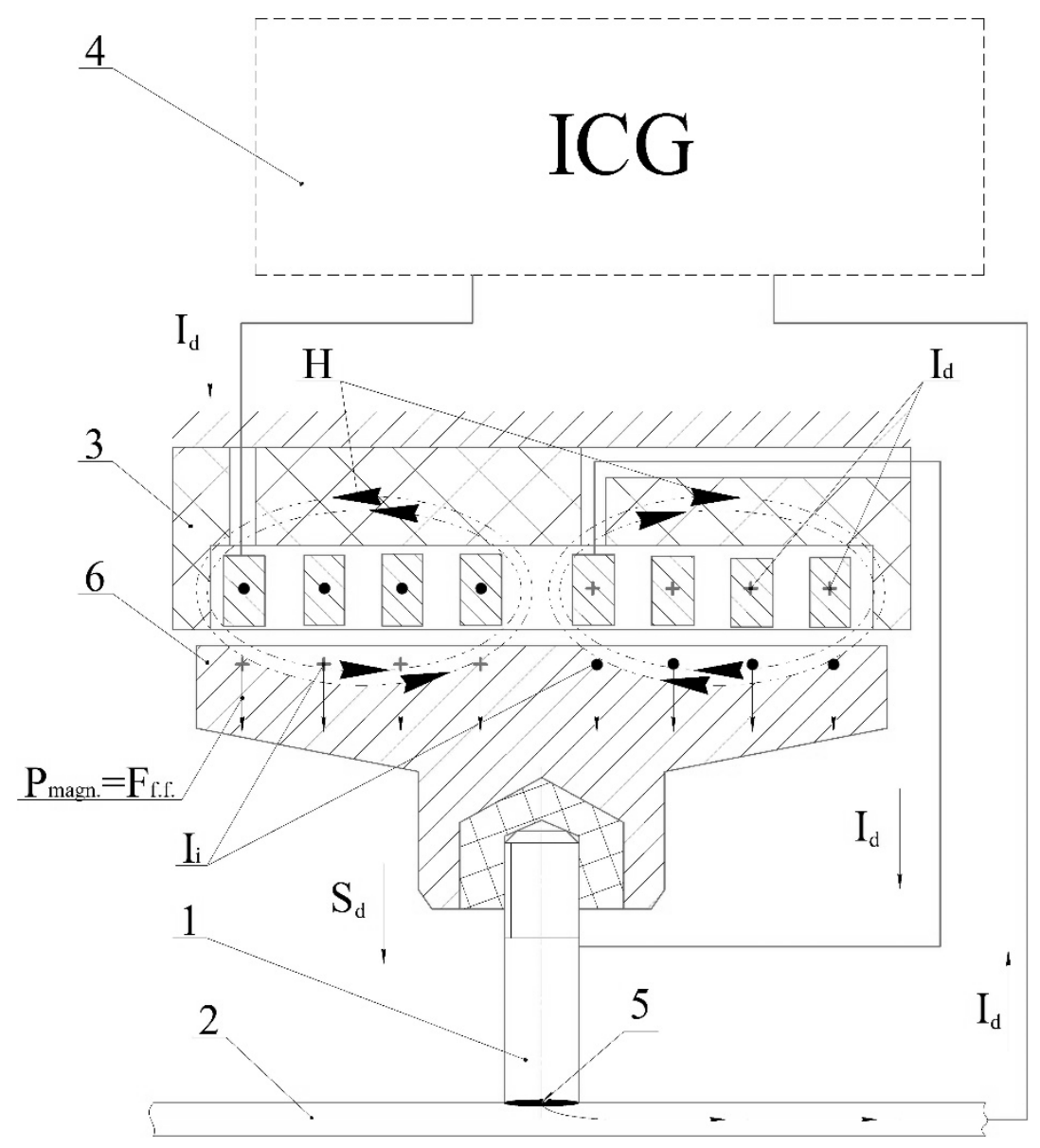

Fig. 3. Basic diagram of CDPW with MPD 1 is core element; 2 is flat part; 3 is MPD inductor; 4 is ICG; 5 is junction zone; 6 is ICG pusher; $H$ is magniflux; $P_{\text {magn. }}$ is magnetic pressure; $F_{\text {f.f. }}$ is forging force; $I_{d}$ is discharge current; $I_{i}$ is induced current; $S_{d}$ is displacement.

The passing current pulse melts the contact surfaces; steam of the molten metal creates a natural protection of the weld pool from the external environment. The forging force transmitted by the fastener allows for a splash of the molten metal together with contaminants (oxide and adsorbed films, non-metallic inclusions) out of the welding zone. The cleaned surfaces are mutually deformed; the weld is formed in solid. The technology options of CDPW with MPD by the example of welding rod elements of $10 \mathrm{~mm}$ in diameter with sheet metal 0,8 mm thick are shown in Fig. 4. 
6

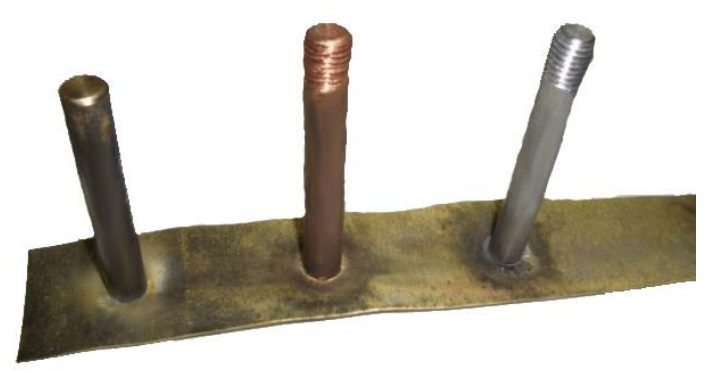

Fig. 4. Experimental sample: L63 sheet steel alloy, core elements from L63, M01 and AMg6 alloys

Three CDPW process flow diagrams are proposed: with electric explosion of the relief, pulsed arc (with preliminary contact or preliminary gap).

As a result of the experimental studies, the following requirements are proposed:

- CDPW with a relief at the end of the core element is preferably used to connect non-ferrous metals with ferrous metals (copper, aluminum, nickel with steel, etc.);

- CDPW with MPD without initial gap should be used for welding aluminum, copper, nickel alloys in a uniform combination;

- CDPW technology with the original gap should be used for welding dissimilar non-ferrous alloys.

Magnetic-pulse welding in vacuum.

It is possible to intensify the diffusion welding of female joints using magnetic-pulse processing according to the «crimp» scheme. As in diffusion welding, the process must be performed with preheating in vacuum to prevent oxidation of the connectable surfaces.

In contrast to the classical magnetic-pulse welding, the pressure is carried out along the normal to the connectable parts; there is no cumulative cleaning jet arising from glancing collisions. Cleaning of surfaces is carried out through the vacuum-thermal preheating. In this case, the magnetic pressure in the weld junction should be uniform $[9,10]$.

The diagram of the device for magnetic-pulse welding in vacuum of emission coatings is shown in Fig. 5 [11].

Under the bulk welding of thin-walled lining coatings (often of an open loop) from materials with low electrical conductivity (these are often metal-alloy emission coatings), the welding was performed by one of the magnetic-pulse processing techniques - the impact was carried out through the transmitting shell - «satellite». Copper-nickel-plated sleeves were used with the wall thickness of $\mathrm{t}_{\mathrm{cm}}=0,210^{-3} \mathrm{~m}$, selected due to the need for non-penetration through the material of the magnetic field $(H)$. 


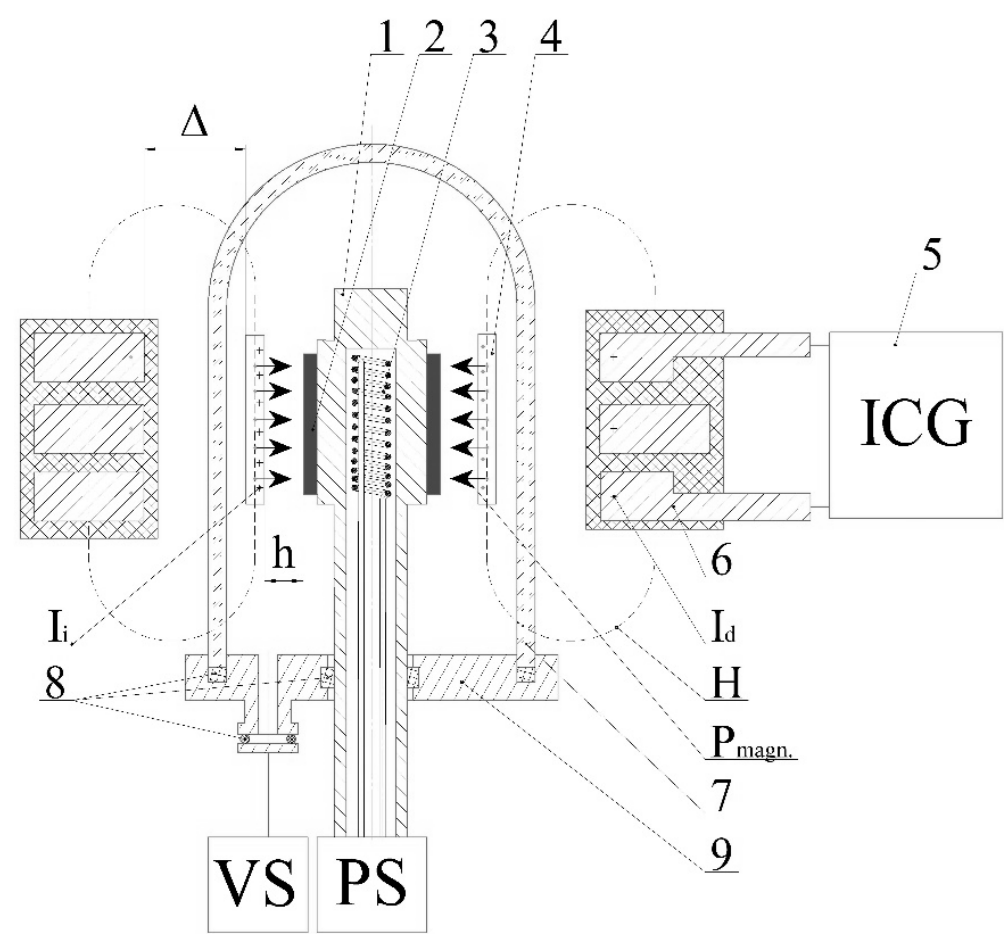

Fig. 5. Diagram of magnetic-pulse welding in vacuum1 base (core); 2 is casing (emitter); 3 is heating element; 4 is satellite; 5 is pulse current generator; 6 is MPD inductor; 7 is quartz glass; 8 is vacuum seal; 9 is vacuum chamber base; $I_{d}$ is discharge current; $H$ is magniflux; $I_{i}$ is induced current; $P_{\text {magn }}$ is magnetic pressure; PS is power source; VS is vacuum system; h is gap between emitter and satellite; $\Delta$ is gap between inductor and shell

The process is as follows. The base (core) (1) with the lining (2) and the satellite (4) are fixed in a vacuum chamber with the heating element (3) in the zone of the inductor magnetic pulse processing. Then, the air is pumped from the vacuum chamber to the pressure of $0,66 \cdot 10-2 \mathrm{~Pa}$. After that, the heating element is turned on, the temperature rises gradually to $(0,8-0,9) \cdot \mathrm{Tm}$ (melting point of the least refractory material) of the prepared materials. Then, the impulse current generator (5) (ICG) is started out and discharged to the work tool-inductor (6).

Under the interaction with the magnetic field of the inductor $(\mathrm{H})$ and the currents (Ii) induced in the satellite, the magnetic pressure (Pmagn.) occurs. It compresses the satellite (4), which affects the emitter (2). Welding is performed in solid.

This device uses a unique feature of the magnetic-pulse welding, a remote effect through a dielectric quartz glass (7), which is a part of the vacuum chamber. The cathode assembly is in vacuum, and the tool - MPD inductor is in the atmosphere.

The magnetic-pulse welding in vacuum with preheating enables to improve welding techniques in solid. The technique allows us to obtain overlap telescopic joints of light-gauge pipes with structural parts from homogeneous and dissimilar materials, lining cylindrical 
8

parts with thin-walled materials at the ratio of the welded strip width to the base diameter $(1 / \mathrm{d}>2)$, and to weld on the "developed" surfaces. In this way, joints of various metals and alloys widely used in the manufacture of electronic equipment were obtained.

Fig. 6 shows the assemblies consisting of molybdenum cores with platinum-barium emitters welded through MPW in vacuum.

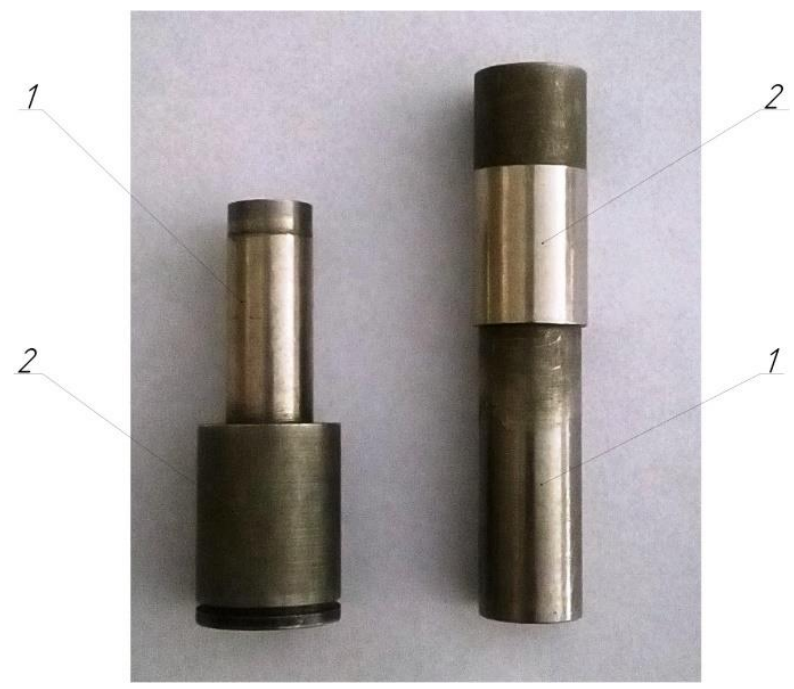

Fig. 6. Weldable cathode assemblies 1 - the core of the cathode; 2 - emitter

\section{Conclusion}

The scientific-technical material on the advanced welding techniques presented in the form of a comprehensive review suggests that Russian researchers and experts successfully continue to develop the magnetic-pulse technologies.

Regardless their limited financial resources, as well as their shortage in the knowledgeintensive industries, Russian research institutions still have significant scientific, technical, production and practice-oriented capabilities in the development and application of the pulsed electrotechnics.

\section{References}

1. V. I. Lysak, S. V. Kuzmin, Machine building, Moscow, 544 p. (2005)

2. G. V. Konyushkov, R. A. Musin, Publ. House IPR Media, 632 p. (2009)

3. E. L. Strizhakov, S. V. Nescoromniy, D. V. Minko, Monography, (Rostov-onDon, DSTU Publ. Centre, 201 p. 2016)

4. E. L. Strizhakov, S. V. Neskoromny, S. O. Ageev, Welding International, 29 (12), pp. 988-990 (2015) 
5. E. L. Strizhakov, M. Yu. Batsemakin, S. V. Nescoromniy, Physics and Chemistry of Materials Treatment, 1, pp. 64-67 (2007)

6. E. L. Strizhakov, N. A. Karandashev, M. Yu. Batsemakin, Patent RF 2228826 (2004)

7. S. V. Nescoromniy, E. L. Strizhakov, Vector of science of Togliatti State University, 2-2 (32- 2) pp. 123-129 (2015)

8. D. M. Kaleko, Autowelding, 10(714), pp. 29-36 (2012)

9. B. E. Paton, D. M. Kaleko, A. R. Bulatsev, V. F. Shulym, Autowelding, 4(708), pp. $42-46$ (2012)

10. S. V. Nescoromniy, E. L. Strizhakov, Patent RF 70839 (2008)

11. E. L. Strizhakov, S. V. Neskoromnyi, R. V. Merkulov, Welding International, 24(9), pp. 718-720 (2010)

12. E. L. Strizhakov, et al., Welding Production, 3, pp. 42-46 (2015)

13. S. V. Neskoromny, E. L. Strizhakov, Vestnik of DSTU, 6(49), pp. $896-899$ (2010)

14. E. L. Strizhakov, S. V. Nescoromniy, S. O. Ageev, Welding Production, 5, pp. 53-58 (2018)

15. V. A. Glushchenkov, V. A. Karpukhin, (Samara: «Fedorov» Publ. House, p. 208 2014)

16. E. L. Strizhakov, A. Yu. Kem, A. I. Bokov, 16th Int. PLANSEE Seminar, 4, pp. 287-293, Reutte/Tirol, Austria (2005) 